\title{
Influence of Wing Elasticity on the Experimental and Numerical Determination of Dynamic Derivatives
}

\author{
A.-R. Hübner, andreas.huebner@dlr.de; \\ T. Kilian, thomas.kilian@dlr.de; \\ F. Spiering, frank.spiering@dlr.de \\ Deutsches Zentrum für Luft- und Raumfahrt (DLR), Institute of Aerodynamics and Flow \\ Technology, Lilienthalplatz 7, D-38108 Braunschweig, Germany
}

\begin{abstract}
Summary
The presented investigations deal with the analysis of dynamic derivatives of a modern transport aircraft configuration (DLR-F12). The conducted wind tunnel tests focused mainly on the measurements of unsteady forces, moments and pressure distributions. The measurements of the steady and unsteady wing deformations using two different wings were of significant importance. Those wings are designed to be ideal stiff on the one hand but also to allow more flexibility on the other hand, in order to investigate the effect of wing elasticity on the static and dynamic aerodynamic properties of this configuration. For the validation of the numerical tools calculations are performed based on the solution of the unsteady Reynolds-averaged Navier-Stokes (URANS) equations using a finite volume parallel solution algorithm with an unstructured discretization concept (DLR TAU-Code). The wing deformation is accounted for using the finite element code ANSYS.
\end{abstract}

\section{Nomenclature}

\begin{tabular}{|l|l|l|l|}
\hline$b$ & wing span, $\mathrm{m}$ & $w$ & wing bending at c/4-chord, mm \\
\hline$V$ & freestream velocity, $\mathrm{m} / \mathrm{s}$ & $\Delta \epsilon$ & wing twist, deg \\
\hline$c_{r}$ & reference chord length, $\mathrm{m}$ & $F_{Z}$ & lift force, $\mathrm{N}$ \\
\hline$\alpha$ & angle of attack, deg & $M_{Y}$ & pitching moment, Nm \\
\hline$\dot{\alpha}$ & angular velocity, 1/s & $c_{L q}+c_{L \dot{\alpha}}$ & lift due to pitch motion \\
\hline \multirow{2}{*}{$\alpha_{0}$} & $\begin{array}{l}\text { mean angle of attack, } \\
\text { (unsteady simulation, deg.) }\end{array}$ & $c_{m q}+c_{m \dot{\alpha}}$ & pitchdamping-derivative \\
\cline { 3 - 4 } & pitching rate, $1 / \mathrm{s}$ & $\mathrm{CSM}$ & Computational Fluid Dynamics \\
\hline$q$ & frequency, $\mathrm{Hz}$ & DOF & Demputational Structure Mechanics \\
\hline$f$ & & \multicolumn{2}{c}{} \\
\hline
\end{tabular}

\section{Introduction}

During the extensive process of aircraft development it is highly desirable to obtain information about the future flight mechanics behavior of an aircraft already at a very early stage. The reliability of the predicted data is of eminent importance with regard to cost effectiveness within the design process. The experimental and numerical determination of dynamic derivatives for transport aircraft configurations 
have been performed at the DLR Institute of Aerodynamics and Flow Technology in co-operation with the Low Speed Wind Tunnel Braunschweig (NWB) of the German - Dutch Wind Tunnels (DNW) for more than a decade. Particularly the elasticity effect of a high aspect ratio wing largely affects the static and dynamic behavior of such a configuration. In order to predict these effects, a new "elastic" wing has been designed and built for the generic DLR-F12 wind tunnel model. Furthermore the numerical investigations are carried out using multidisciplinary codes with coupled aerodynamic-structural solvers to address all requirements necessary for an accurate prediction of the unsteady aerodynamics of a maneuvering aircraft.

\section{Experimental Method}

\subsection{Test facility}

The low speed wind tunnel DNW-NWB is operated by the foundation "German Dutch Wind Tunnels" and is located on the DLR site in Braunschweig, Germany. It's an atmospheric, closed circuit facility with a test section size of $3.25 \mathrm{~m} \times 2.80 \mathrm{~m}$. It can be operated with an open, closed or slotted test section. The maximum free stream velocity is $80 \mathrm{~m} / \mathrm{s}$ in the closed test section and $70 \mathrm{~m} / \mathrm{s}$ in the open test section. More information about the DNW-NWB can be found in Kausche et.al. [1].

\subsection{Wind tunnel model}

The DLR-F12 model used is a typical geometry of a generic transport aircraft and was constructed specifically for dynamic tests in the DNW-NWB. Such a model must meet different design criteria than conventional wind tunnel models. It has a span of $b=2.036 \mathrm{~m}$, a reference chord of $c_{r}=0.253 \mathrm{~m}$ and a fuselage length of $l=2.238 \mathrm{~m}$. It is made of a carbon fiber sandwich construction, having a weight of approximately $12 \mathrm{kgs}$. In order to evaluate the influence of individual components of this configuration the model is designed in a modular way, such that every component of interest can be added. Two different wings exist for the DLR-F12 model. The standard "stiff wing" of the DLR-F12 model has been designed for maximum stiffness, low weight and high Eigenmodes. The geometry corresponds to the 1-g flight shape of a typical transport aircraft wing. Under operating condition $\left(\alpha=8^{\circ}\right.$, $V=70 \mathrm{~m} / \mathrm{s}$ ) the static bending displacement at the tip is $8 \mathrm{~mm}$ while the twist at the wing tip is $0.4^{\circ}$. The other wing is labeled the "elastic wing". As the name implies, it has a significantly lower stiffness than the "stiff wing". The "elastic wing" was designed and built to investigate the effect of wing elasticity on the static and dynamic aerodynamic properties of the DLR-F12 configuration. It ideally has to maintain the weight and Eigenmode properties of its stiff counterpart, while at the same time possessing a twist and bending distribution similar to a distribution of a comparable full size aircraft. The "elastic wing" is designed in a way that it's deformed shape at $\alpha=4^{\circ}$ and $V=70 \mathrm{~m} / \mathrm{s}$ coincides with the shape of the "stiff wing". Without any aerodynamic loads acting on the "elastic wing" its shape is consistent with the jig shape of a conventional wing (see Fig. 1). The designed maximum deformations of the "elastic wing" is $5,7^{\circ}$ of twist and $63 \mathrm{~mm}$ of bending at the wing tip $\left(\alpha=8^{\circ}\right.$, $V=70 \mathrm{~m} / \mathrm{s})$. 


\subsection{Model support structure}

For the experimental determination of the dynamic wind tunnel data a new combined motion test capability was developed by DNW and DLR as an improved successor to the previous test set-ups, using a unique 6-DOF test rig called "Model Positioning Mechanism" (MPM). This novel test facility (see Fig. 2 shown with the DLR-F12 model installed), based on a parallel kinematic concept, was integrated into NWB's wind tunnel environment in 2004. With its large amplitude (up to $\pm 15^{\circ}$ in pitch) and high rate (up to $f_{0}=3 \mathrm{~Hz}$ ) arbitrary motion the mechanism is well adapted for dynamic wind tunnel tests. More details concerning the MPM can be found in [2], [3], and [4].

\subsection{Model instrumentation}

The internal model instrumentation consists of a 6 component strain gauge balance, type Emmen 196-6, and a Lucas-Schaevitz inclinometer for static measurement of the angle of attack. The pressure ports are arranged in three onflow-parallel sections on wing, horizontal and vertical tail plane. The "elastic wing" has an additional pressure measurement section on the left hand side of the wing, mirroring the one on the right hand side at the symmetry plane. The pressure taps are connected to 32-port PSI pressure measurement modules, which are located inside the model. All connecting tubes are of same length. Additionally two to four Kulite pressure transducers have been installed in situ in very close proximity to the other pressure taps. The Kulite transducers are used as reference sensors for the dynamic measurements. The dynamic data of the multiport pressure measurement modules have been corrected with respect to attenuation and phase shift according to [5].

\subsection{Model position and deformation measurement}

Static and dynamic model positions as well as the deformation of the left wing have been measured with two optical systes. A 3D system consisting of two Mikrotron Mc1310 video cameras is used to measure the wing deformation by recording images of two rows of markers which are applied to the model's surface. And an additional video system with a single Pulnix high speed video camera has been used to measure the nominal instantaneous attitude by observing two markers on the fuselage. This system has been used for position measurement during several dynamic tests at the DNW-NWB [2], [5], [6].

The cameras of the 3D system have a resolution of $1280 \times 1024$ pixels; a frame rate of 300 images per second has been used. The cameras have been installed outside the test section below the test section floor, looking through glass windows, which can be seen in the complete set-up inside the test section, shown in Fig. 3. The light source for illumination of the markers, which is accentuated in this picture, consists of an array of 4500 low cost LEDs emitting light of $400 \mathrm{~nm}$ wave length (near ultra violet or black light). The markers are applied on the model surface with commercially available fluorescent paint. Operating the LED at a pulse rate which coincides with the frame rate of the camera system and using a special set of filters leads to camera images completely void of reflections. For deformation 
measurements 16 markers have been applied to the upper side of the left wing, arranged in two rows parallel to the leading edge and the trailing edge, as can be seen in Fig. 4. The wing root close to the fuselage and the central fuselage are considered to be stiff enough to be used for the models reference attitude. After calibration of the cameras the camera control software picCOLOR9 yields $\mathrm{x}, \mathrm{y}$ and $\mathrm{z}$ co-ordinates for every marker. The processing of the marker co-ordinates towards twist and bending distributions is done by software written as part of a diploma thesis [7]. Similar to the determination of dynamic derivatives, wind-off values are subtracted from wind-on values to calculate the aerodynamic induced deformation [6].

\section{Numerical Method}

\subsection{CFD Solver DLR TAU-Code}

The flow around the aircraft configuration is simulated with the DLR TAU-Code which solves the compressible, time-accurate Reynolds-Averaged Navier-Stokes equations using a finite-volume formulation (see [8]) with the Spalart-Allmaras turbulence model. The grids were created with the commercial grid generator Centaur. All surfaces of the model are considered as viscous walls on which 30 layers of prismatic elements are used in order to resolve the turbulent boundary layers around the configuration. A maximum boundary layer resolution of $y^{+}=0,85$ was reached. The remaining part of the computational domain is discretized with tetrahedral elements resulting in a grid containing about $8,67 \times 10^{6}$ grid points overall. The radius of the spherical farfield is $r=50 \mathrm{~m}$. The inviscid fluxes are calculated by employing a central method with scalar dissipation, which is used for the present study. The gradients of the flow variables are determined with a Gauss-Green formula. The viscous fluxes are discretized using central differences. For time-accurate computations a dual-time stepping approach for 1st-, 2nd- or 3rd-order discretization in physical time is implemented in TAU. An explicit multistage Runge-Kutta time stepping scheme is used for steady calculations. For time accurate computations, an implicit time stepping approach (LU-SGS scheme) is used. The initialisation of the unsteady computation was performed with converged steady computations in order to reduce the CPU time needed to obtain the physical unsteady flow. The simulation included 2 periods with a resolution of 50 physical time steps per period.

\subsection{Fluid-structure coupling}

In order to take aeroelastic effects into account, the numerical simulations included a recently developed in-house process scheme for iterative fluid-structure coupling. It consists of a CFD solver (DLR TAU-Code), a CSM solver (ANSYS), a grid deformation module and a coupling module to transfer the data between the previously named components. Except for the CSM solver all major components of the scheme can be run in parallel, which leads to a significant reduction in computation time. The first step of the procedure is the computation of the aerodynamic loads on the grid of the current coupling step. In the first coupling step the undeformed initial 
grid is used. Afterwards the loads from CFD are mapped onto CSM coupling surface and the structural deformation on the finite element model is computed. The deformation from CSM is mapped onto CFD coupling surface and a new volume mesh via deformation of the current one is created. In the following coupling step this new mesh is used to compute aerodynamic loads and the flow data obtained in the last coupling step is used as restart to accelerate the convergence of the computation. The final shape of the aeroelastic equilibrium is obtained when the loads of two consecutive coupling steps become equal.

\section{Results}

\subsection{Results from static tests}

The FE model used for the coupled computations was provided by the engineering office "Leichtwerk" as part of the structural design of the "elastic" wing. It matches the predefined jig-shape and its structural properties were calculated using the FE-code ANSYS as well as an in-house vortex lattice method. In order to validate the FE model the predicted wing deformation at a set of design load cases was compared to the experimental data. The 4 load cases investigated are at a freestream velocity of $V=70 \mathrm{~m} / \mathrm{s}$ and angles of attack ranging from $\alpha=-5^{\circ}$ to $\alpha=8^{\circ}$. The comparison of static wing bending $w$ as well as twist $\Delta \epsilon$ values, depicted in Fig. 5, yields a good agreement of the experimental as well as the numerical resluts and the design data. Merely the spanwise twist distributions show a slight deviation of bending and twist of 5 to $7 \%$ at the wing tip at $\alpha=8^{\circ}$. A comparison of the wing bending and twist at a position close to the tip at three freestream velocities is shown in Fig. 6. In the region of constant lift-slope a high consistency of the experimental and CFD data is revealed. Only a slightly lower gradient of the numerical curves can be observed towards higher velocities. At higher angles of attack at $V=35 \mathrm{~m} / \mathrm{s}$ and $56 \mathrm{~m} / \mathrm{s}$ the numerical simulation underestimates the loss of lift due to separation. This decrease in lift becomes manifest in a decrease in bending as well as a slight decrease in negative twist. The unsteadiness of the twist curves results from the small chord length a the tip, which leads to a larger impact of the measurement accuracy.

\subsection{Results from dynamic tests}

Sinusoidal pitch oscillations have been performed with an amplitude of $\Delta \alpha=4^{\circ}$ and oscillation frequencies of $f_{0}=3 \mathrm{~Hz}$ at a freestream velocity of $V=70 \mathrm{~m} / \mathrm{s}$. Dynamic derivatives can be derived by Fourier analysis from the time signals of the position, forces and moments. The time history as well as hysteresis curves for the vertically acting force $F_{Z}$ and the pitching moment $M_{Y}$ are shown in Fig. 7. Comparable to the experimental data using the "stiff" wing the numerical calculations were done with the same amplitude of $\Delta \alpha=4,52^{\circ}$. However the maximal allowable amplitude of the "elastic" wing within the measurement is only $\Delta \alpha=4^{\circ}$, but the effects of the amplitude on the derivatives is not significant. The backward swept wing geometry leads to a negative twist when bended upwards, which causes a lift reduction. The wing elasticity amplifies this effect. This explains the overall higher amplitudes 
noticeable for the stiff wing. The different gradients of the hysteresis curves can also be explained by this aeroelastic effect, which correspond to the static derivatives shown in Fig. 8. The absolute values for the "elastic" wing are $15 \%$ lower than those for the "stiff" wing. The influences on the dynamic derivatives are almost in the same manner. The lift derivative and the pitch damping decrease up to $13 \%$ and $7 \%$ respectively. These trends are similar to the depicted experimental data. Only the dynamic lift derivativ, which is extremely sensitive to phase deviations [3], is numerically underestimated. This deviation might be a result of higher harmonics in the signals. The evaluation process however is based on the first harmonic of the oscillation frequency.

\section{Conclusions}

In order to quantify the effect of wing elasticity on the prediction of the static and dynamic behavior of an aircraft a new "elastic" wing was built for the DLR-F12 wind tunnel model. Systematic experimental investigations have been performed with the model using the MPM model support for dynamic tests in the DNW-NWB. The research included measurements of force, surface pressure and wing deformations for static as well as dynamic cases. The comparison of the experimental data with the first numerical solutions, which are gained using multidisciplinary codes with coupled aerodynamic-structural solvers show significant discrepancies of the static and dynamic derivatives obtained with the "elastic wing" and the "stiff wing". Therefore aeroelastic effects have to be accounted for in order to accurately predict the dynamic derivatives and further numerical calculations are necessary for the validation process of the TAU code.

\section{References}

[1] Kausche, G., Otto, H., Siebert, R. and Christ, D. The Low-Speed Wind Tunnel at DFVLR in Brunswick (Status 1988), DFVLR-Mitt. 88-25 (1988).

[2] Loeser, T., Bergmann, A.: Development of the Dynamic Wind Tunnel Testing Capabilities at DNW-NWB. AIAA-2003-453, Reno, Nevada, (2003).

[3] Bergmann, A., Hübner, A.-R., Löser, T.: Experimental and numerical research on the aerodynamics of unsteady moving aircraft. Elsevier Journal, Progress in Aerospace Sciences, Vol. 44, pp 121-137, (2008).

[4] Bergmann, A. et al.: "MPM" USA Patent Application. Pub. Npo. US 2006/0254380 A1. (2006).

[5] Hübner, A.-R., Bergmann, A., Loeser, T.: Experimental and Numerical Investigations of Unsteady Force and Pressure Distributions of Moving Transport Aircraft Configurations. AIAA-2009-91, Orlando, Florida, (2009).

[6] Hübner, A.-R.: Experimentelle und numerische Bestimmung der dynamischen Derivative. Dissertation, DLR-FB 2009-26, Braunschweig, (2009).

[7] Kilian, T.: Einfluss der Flügeldeformation der DLR-F12 Konfiguration bei der experimentellen und numerischen Ermittlung der statischen und dynamischen Derivative. Diplomarbeit Nr. 02/2008, DLR-AS (2009).

[8] Schwamborn, D.; Gerhold T.; Heinrich R.: The DLR TAU-Code: Recent Applications in Research and Industry, TU Delft, Delft, Netherland, 2006. 


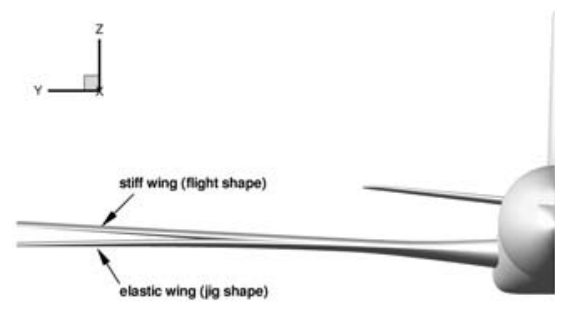

Figure 1: DLR-F12 model deformation of the stiff and elastic wing

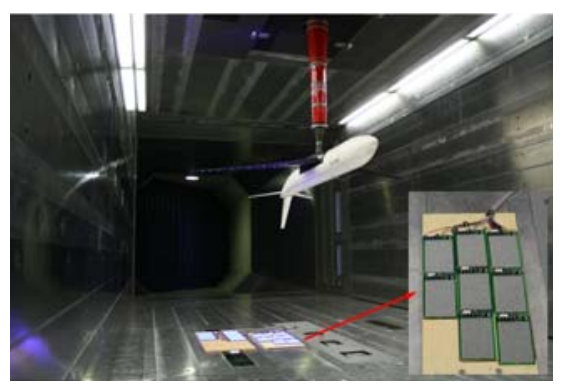

Figure 3: DLR-F12 model illumination with UV LEDs

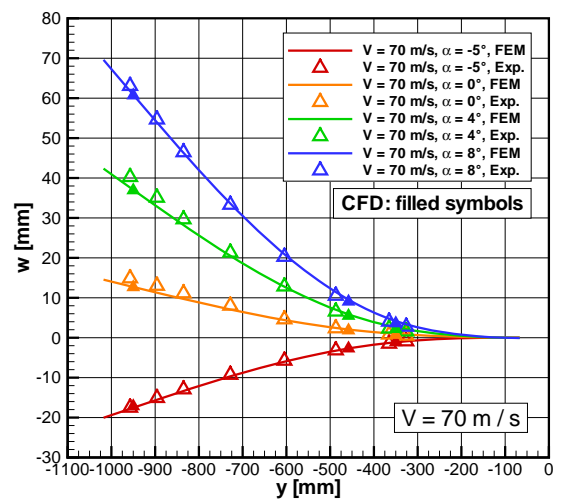

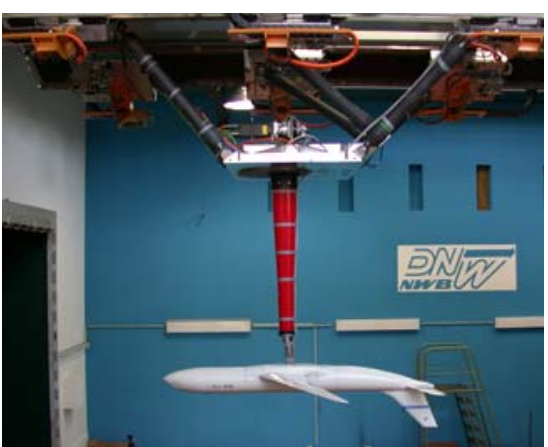

Figure 2: DLR-F12 model on the MPM in the DNW-NWB

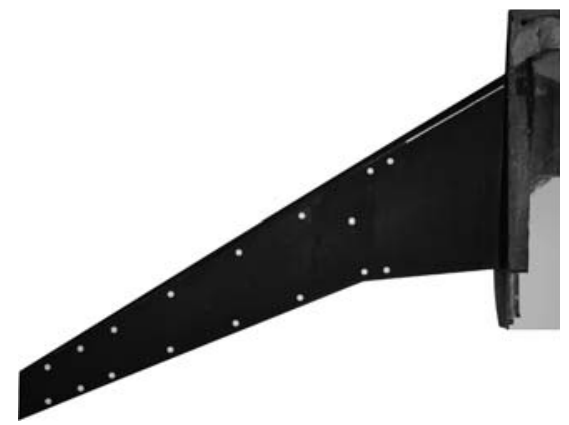

Figure 4: Markers on the DLR-F12 wing

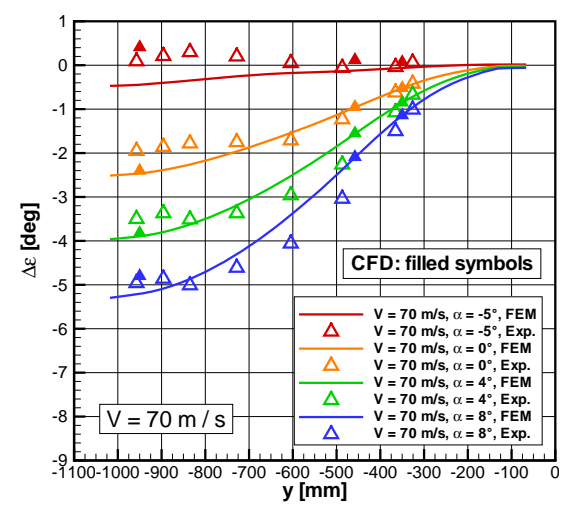

Figure 5: DLR-F12, elastic wing, bending and twist distribution in spanwise direction for different AoA, comparison of experimental / numerical results and design data 

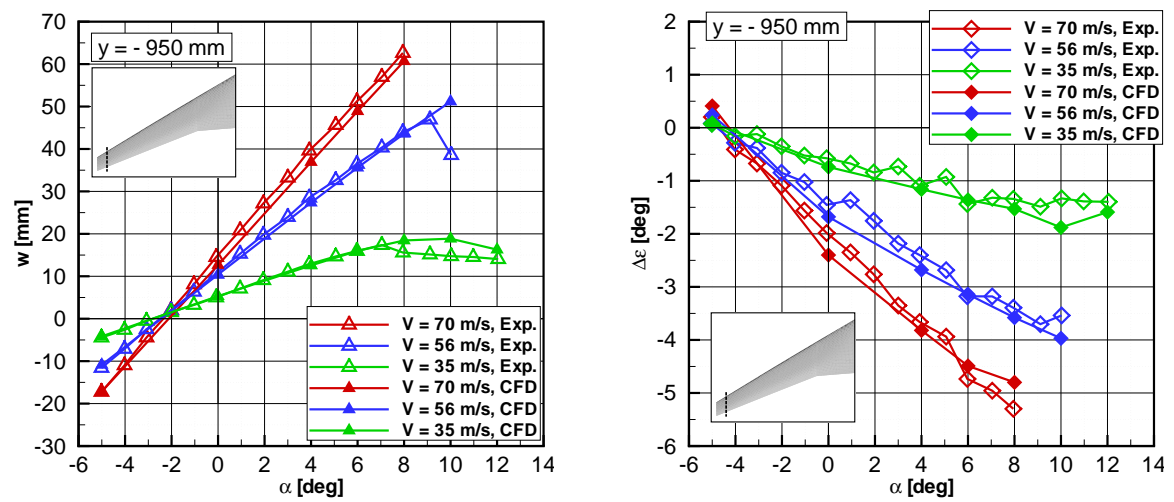

Figure 6: DLR-F12, elastic wing, bending and twist distribution of a wing section for different velocities, comparison of experimental and numerical results
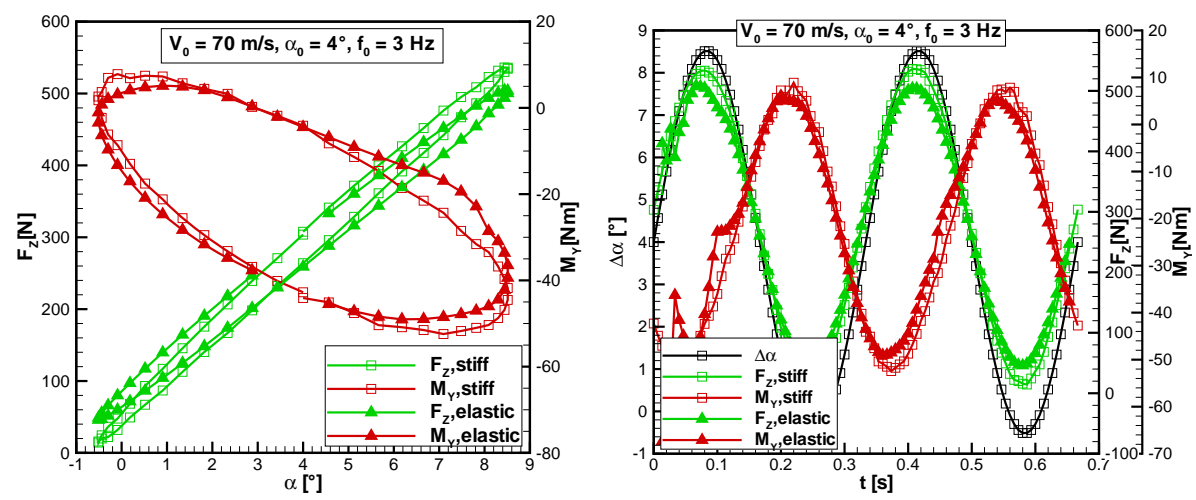

Figure 7: DLR-F12 model with stiff and elastic wing, numerical results for a pitching oscillation, $\mathrm{U}=70 \mathrm{~m} / \mathrm{s}, f_{0}=3 \mathrm{~Hz}$
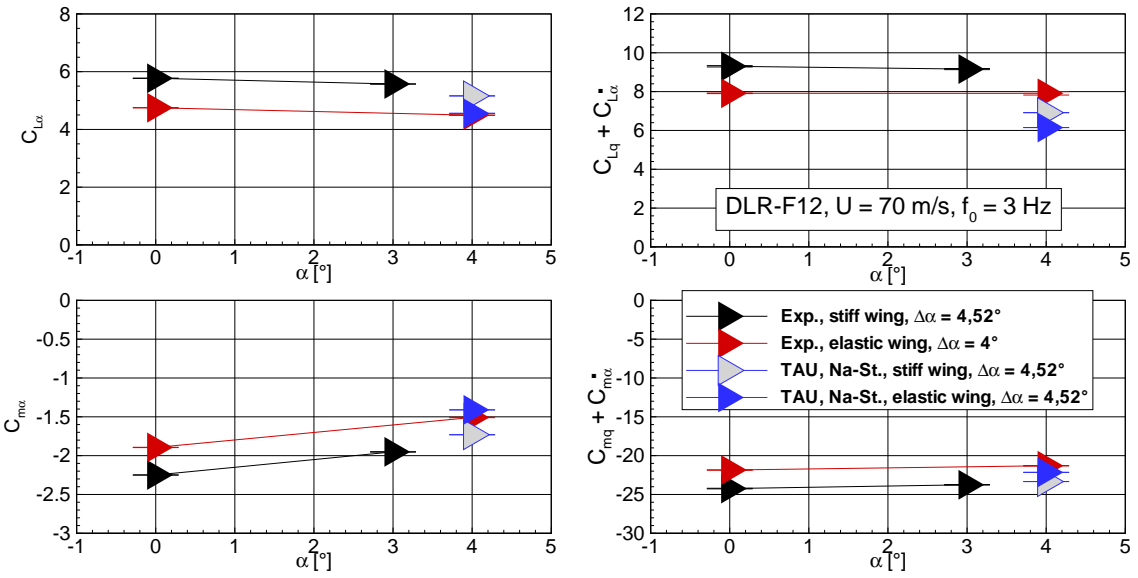

Figure 8: DLR-F12 model, influence of wing elasticity on the derivatives of the pitching motion, comparision of experimental and numerical results 